\title{
Quasi first-principles Monte Carlo modeling of energy dissipation by low-energy electron beams in multi-walled carbon nanotube materials
}

\author{
Dimitris Emfietzoglou, ${ }^{1, a)}$ Ioanna Kyriakou, ${ }^{1}$ Rafael Garcia-Molina, ${ }^{2}$ Isabel Abril, ${ }^{3}$ \\ and Kostas Kostarelos ${ }^{4}$ \\ ${ }^{1}$ Medical Physics Lab, University of Ioannina Medical School, 45110 Ioannina, Greece \\ ${ }^{2}$ Departamento de Física - CIOyN, Universidad de Murcia, E-30100 Murcia, Spain \\ ${ }^{3}$ Departament de Física Aplicada, Universitat d'Alacant, E-03080 Alacant, Spain \\ ${ }^{4}$ Nanomedicine Lab, Centre for Drug Delivery Research, UCL School of Pharmacy, \\ University College London, London WCIN 1AX, United Kingdom
}

(Received 24 December 2011; accepted 5 February 2012; published online 2 March 2012)

\begin{abstract}
The energy dissipation pattern of low-energy electron beams $(0.3-30 \mathrm{keV})$ in multi-walled carbon nanotube (MWCNT) materials is studied by Monte Carlo simulation taking into account secondary-electron cascade generation. A quasi first-principles discrete-energy-loss model deduced from a dielectric response function description of electronic excitations in MWCNTs is employed whereby both single-particle and plasmon excitations are included in a unified and self-consistent manner. Our simulations provide practical analytical functions for computing depth-dose curves and charged-carrier generation volumes in MWCNT materials under low-energy electron beam irradiation. (C) 2012 American Institute of Physics. [http://dx.doi.org/10.1063/1.3688307]
\end{abstract}

Recent work on the irradiation of carbon nanotubes (CNTs) by energetic charged particles has unambiguously revealed various beneficial effects towards beam-assisted engineering of CNT-based nanodevices with the desired properties. ${ }^{1,2}$ Scanning electron microscopy (SEM) and electron-beam lithography (EBL) are increasingly being used for the characterization and fabrication of CNT-based field-effect-transistors ${ }^{3-5}$ and stimulated field-emitters. ${ }^{6-9}$ Since electron transport plays a fundamental role in the ultimate performance of these techniques, knowledge of the energy dissipation pattern of low-energy electron beams $(0.3-30 \mathrm{keV})$ in CNT materials becomes of prime importance. Monte Carlo (MC) simulations offer a valuable tool for investigating energy-transfer phenomena in irradiated solids. ${ }^{10,11}$ In the present energy range, energy dissipation in matter by electron beams is almost exclusively due to inelastic electron-electron scattering. Elastic electron scattering by target nuclei, well-known to be responsible for irradiation damage via knock-on atomic displacement at high beam energies (above about $80 \mathrm{keV}$ for CNTs), ${ }^{12,13}$ results in significant momentum transfer (or equivalent, angular deflection) but practically zero energy loss. ${ }^{14}$

Contrary to the continuous energy-loss models (e.g., from stopping power theory) widely used for studying irradiation effects in bulk solids, MC models of materials with restricted dimensions (e.g., CNTs and nanodevices in general) must account for secondary-electron cascade generation through the use of discrete (or single-scattering) energy-loss models. ${ }^{15-17}$ Such models will also complement current computational studies of high-energy electron-beam (e.g., from a transmission electron microscope, TEM) irradiation effects in CNTs lying on substrates from backscattered electrons. ${ }^{18,19}$

Binary collision theory has been widely used in this context due to its computational convenience, despite its well-

${ }^{\text {a) Electronic mail: demfietz@cc.uoi.gr. }}$ known simplistic description of the materials excitation properties. ${ }^{20}$ In the present work, we advance a MC model of electron-beam energy dissipation in multi-walled carbon nanotube (MWCNT) materials based on a quasi firstprinciples discrete-energy-loss model deduced from a realistic description of the target electronic excitations. This approach has the advantage that secondary-electron cascade generation can be explicitly simulated without the need for an arbitrary separation of plasmon and single-particle losses, since the complete excitation spectrum of MWCNTs is builtinto the model via the energy $(\hbar \omega)$ and momentum ( $\hbar k)$ dependent dielectric response function, $\varepsilon(\omega, k)$. Then, under the constraint of physically motivated sum-rules (which preserve causality), the energy losses in single inelastic collisions can be computed in a self-consistent manner according to the properties of the so-called energy-loss-function (ELF), $\operatorname{Im}[-1 / \varepsilon(\omega, k)]$.

Among several approaches ${ }^{21-24}$ for modeling charged particle induced electronic excitations in CNTs, the opticaldata method ${ }^{25}$ is perhaps most convenient for MC simulation $^{26}$ since it allows important energy-loss magnitudes to be expressed in useful analytic forms ${ }^{27,28}$ with direct use of available experimental data for CNTs. ${ }^{29}$ Therefore, we here employ a many-pole plasmon model of electronic excitations in MWCNTs that permits, within the plane-wave Born approximation (PWBA), the calculation of differential and total inelastic electron-electron scattering cross sections from first-principles. ${ }^{29,30}$ Model parameters associated with the energy, damping rate, and strength of the various excitation modes of the target are determined from spectroscopy data (see Ref. 30 and references therein) under the perfectscreening and Thomas-Reich-Kuhn sum-rule constraints, thus ensuring a realistic and self-consistent description of the electronic excitation properties of MWCNTs over the whole $\omega-k$ plane. To go beyond the standard bulk models of particle-solid interaction or the local dielectric models often used for nanostructures, ${ }^{31}$ dimensionality effects are 
explicitly considered in the model via the analytic extension of $\varepsilon(\omega, k)$ at $k \neq 0$.

The theoretical framework of the PWBA, whereby the incident and scattered particle is represented by plane waves while the interaction is treated by first-order perturbation theory, is particularly convenient since the transition matrix elements are independent of the projectile, and therefore they can be represented by a target-material excitation function such as the ELF used in the present work. However, computing the electron inelastic mean free path within PWBA entails a double quadrature of the ELF over the $\omega-k$ plane that can be impractical for MC modeling over a wide energy range. A convenient parameterization of the PWBA is provided by the Bethe asymptotic expansion. ${ }^{32}$ Such a parameterization for the case of CNTs has been presented in Ref. 33 for both electron and proton projectiles. Along these lines we express here the electron inelastic cross section per unit mass or, equivalent, the density $(\rho)$ normalized inverse inelastic mean free path, $\lambda^{-1} / \rho$, of MWCNT materials as a function of electron energy, $T$ (in $\mathrm{keV}$ ), as follows:

$$
\frac{\lambda^{-1}}{\rho}=\mathrm{const} \frac{\beta \ln (\gamma T)}{T},
$$

where const $=1.63 \times 10^{7} \mathrm{~cm}^{2} / \mathrm{g}$ and $\beta, \gamma$ are material excitation parameters defined through integrals of the ELF; specifically, $\beta$ is obtained from the $\omega$-dependence of the ELF at $k=0$ whereas $\gamma$ also depends upon the properties of the ELF at $k \neq 0$ (see Ref. 33 for more details). For MWCNTs we have $\beta=0.0279 \mathrm{keV}$ and $\gamma=58.8 \mathrm{keV}^{-1}$. However, the Bethe asymptotic expansion up to order $T^{-1}$, as offered by Eq. (1), cannot account for the so-called inner-shell effects that, due to their $T^{-2}$ dependence, become important at low particle velocities. Thus, Eq. (1) provides a good representation (to a few \%) of the PWBA calculations only above $\sim 500 \mathrm{eV}$. ${ }^{33}$ Since inner-shell effects are automatically included in the PWBA (to all orders in $1 / T$ ), we can, in principle, improve the performance of Eq. (1) below $0.5 \mathrm{keV}$ by using further terms in the Bethe asymptotic expansion as, for example, it is done in the Tanuma-Powell-Penn formula. ${ }^{28}$ Alternatively, we have chosen here to fit the ratio $\lambda_{\text {Bethe }}^{-1} / \lambda_{\text {PWBA }}^{-1}$, where $\lambda_{\text {Bethe }}^{-1}$ is given by Eq. (1), by the function $J(T)=(1-a T) /(b-c T)$ with $a=35.4 \mathrm{keV}^{-1}$, $b=-0.7655$, and $c=31.7 \mathrm{keV}^{-1}$. As can be seen from Fig. 1, multiplication of Eq. (1) by the correction function $J(T)$ results in very good agreement (better than $\pm 3 \%$ ) with the numerical PWBA results ${ }^{33}$ down to $50 \mathrm{eV}$ (the present simulation cut-off).

For the elastic scattering, calculations are based on the semi-empirical atomic model of Browning ${ }^{34}$ which employs a modified form of the screened Rutherford cross section that approximates analytically basic trends of partial wave calculations of the Mott elastic scattering cross section. ${ }^{20}$ The Browning model is particularly convenient for MC simulation since elastic scattering cross sections can be implemented in a manner similar to the screened Rutherford model. It can be seen from Fig. 1 that for not too low electron energies and up to the maximum electron energy of interest here $(30 \mathrm{keV})$, the Browning model compares fairly well with the corresponding NIST (Ref. 35) values for car-

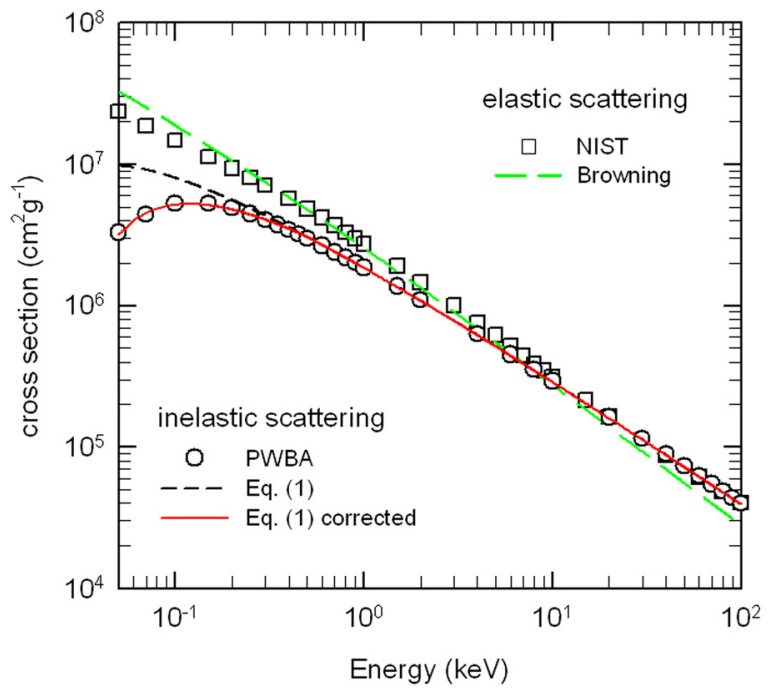

FIG. 1. (Color online) Cross section (per unit mass) for elastic and inelastic electron scattering in MWCNT materials as a function of electron energy. The PWBA and NIST results for comparison are from Refs. 33 and 35, respectively.

bon, which are derived from elaborate partial wave calculations. ${ }^{36}$

The discrete energy losses in inelastic electron-electron scattering were determined from the differential inverse IMFP, $d \lambda^{-1} / d \omega$, which in the PWBA reads

$$
\frac{d \lambda^{-1}}{d \omega}=\frac{\hbar}{\pi a_{0} T} \int_{k_{-}}^{k_{+}} \operatorname{Im}\left[-\frac{1}{\varepsilon(\omega, k)}\right] \frac{d k}{k}
$$

with the limits of integration being $k_{ \pm}=(\sqrt{2 m} / \hbar)$ $(\sqrt{T} \pm \sqrt{(T-\hbar \omega)})$, where $m$ is the electron mass. Since the inverse dependence on $k$ is much stronger than the $k$-dependence of the ELF, the procedure for simulating the discrete energy losses via Eq. (2) may, to a first approximation, be simplified by working in the so-called optical approximation, i.e., by sampling $\operatorname{Im}[-1 / \varepsilon(\omega, k=0)]$, thus avoiding the $k$ integration. We have found that significant improvement can be achieved if we disperse the ELF using an "effective" wavenumber $\left(k_{\text {eff }}\right)$ the magnitude of which is of the same order as $k_{-}$. Specifically, by setting $k_{e f f}=2 k_{-}$we have confirmed that the $k_{\text {eff }}$ approximation reproduces the energy loss rate of the PWBA to within 5\% over the whole energy range of interest here. By this approximation we retain (to a good degree) the correct shape of $d \lambda^{-1} / d \omega$ while still avoiding the numerical integration in Eq. (2). The required extension of the ELF to finite wavenumbers is evaluated from a bulk planar surface (BPS) model ${ }^{30}$ which accounts for boundary effects in the spatial dispersion of MWCNT electronic excitations.

One of the merits of the present formalism is that both single-particle and plasmon excitations are considered selfconsistently within one model. Specifically, we allow for the excitation of plasmons whenever the energy transfer, $\hbar \omega$, is within $E_{p l} \pm$ HWHM, where $E_{p l}=\hbar \omega_{p l}$ and HWHM are, respectively, the energy and half-width-at-half-maximum of the $\pi+\sigma$ plasmon peak. However, plasmons are allowed to decay to single-particle excitations via the Landau damping 
mechanism by giving all their energy to a single target elec$\operatorname{tron}^{37}$ whenever the effective wavenumber is above the cutoff value, $k_{\text {cut }} \approx \omega_{p l} / v_{\mathrm{F}}\left(v_{\mathrm{F}}\right.$ being the Fermi velocity). On the other hand, energy losses outside the plasmon channel are assumed to produce single-particle excitations in the form of either localized electron-hole pairs (for $\hbar \omega<E_{p l}-$ HWHM) that deposit their excitation energy "on the spot," or ionizations (for $\hbar \omega>E_{p l}+$ HWHM). In the latter case, secondary electrons are simulated with kinetic energy equal to the energy transfer minus the binding energy $(B)$ of the ionized shell. For the K-shell, we use the K-edge of carbon, $B_{\mathrm{K}}=285 \mathrm{eV}$, whereas for the valence-shells we use the optical-data threshold, $B_{\mathrm{val}}=3 \mathrm{eV}$; the exact values are inconsequential here. Following K-shell ionization, it is assumed that an Auger electron is emitted isotropically with energy $T_{\text {Auger }}=B_{\mathrm{K}}-2 B_{\text {val }}$. All secondary electrons are simulated in the same manner as the primary electrons.

In principle, the ELF can be used to also sample the inelastic scattering angles through $d^{2} \lambda^{-1} / d \omega d k$. However, since the contribution of inelastic scattering to the angular deflection of the electron beam is relatively small compared to elastic scattering, and in order to avoid the formidable computational task of handling bidimensional tables of ELF, we adopt the simple Moller expressions $\sin ^{2} \theta_{\text {prim }}=2 W_{r} /$ $\left(2+\mu-\mu W_{r}\right)$ and $\sin ^{2} \theta_{\sec }=2\left(1-W_{r}\right) /\left(2+\mu W_{r}\right)$ with $\theta_{\text {prim }}$ and $\theta_{\text {sec }}$ being the scattering angles of the primary and secondary electrons, respectively, and $W_{r}=\hbar \omega / T$, $\mu=T / m c^{2}$.

The use of a discrete-energy-loss MC model permits an event-by-event simulation of the irradiation process, that is, the sequential simulation of each elastic and inelastic collision as the beam electrons (and all generations of secondary electrons) slow down in the irradiated material. The outcome of an event-by-event simulation is, among other things, a set of Cartesian coordinates $\left\{\left(x_{i}, y_{i}, z_{i}\right) ; i=1, \ldots, n\right\}$ of the $n$-number of collisions in a random electron track. In all simulations carried out here the transport of electrons is stopped once their energy falls below $50 \mathrm{eV}$. A low-energy cut-off is necessary to satisfy the general restriction of the PWBA that the projectile is fast compared to the target electrons. Results are average values over 100000 primary (beam) electrons to ensure a small statistical uncertainty (generally less than $1 \%)$.

In Fig. 2 we present MC transport simulations of different measures of the distances traveled by the electron beam in MWCNT materials over the $0.3-30 \mathrm{keV}$ energy interval. The results are density-normalized to facilitate their use for MWCNT materials of different densities. In the main panel we present the electron pathlength, absorption depth, and maximum penetration depths both perpendicular (laterally) and along (axially) the incident direction. The electron pathlength is defined as the average value of the total distance traveled (i.e., of the actual tortuous path) by a primary (beam) electron until its energy falls below the simulation cut-off. If we designate by $\vec{r}_{i}$ the vectors that connect the interaction points with Cartesian coordinates $\left(x_{i-1}, y_{i-1}, z_{i-1}\right)$ and $\left(x_{i}, y_{i}, z_{i}\right)$, then the average value of $r_{\text {total }}=\sum_{i=1}^{n}\left|\vec{r}_{i}\right|$ defines the electron pathlength. For the calculation of the absorption depth as well as the maximum penetration depths (axially or laterally) we first identify the incident beam direc-

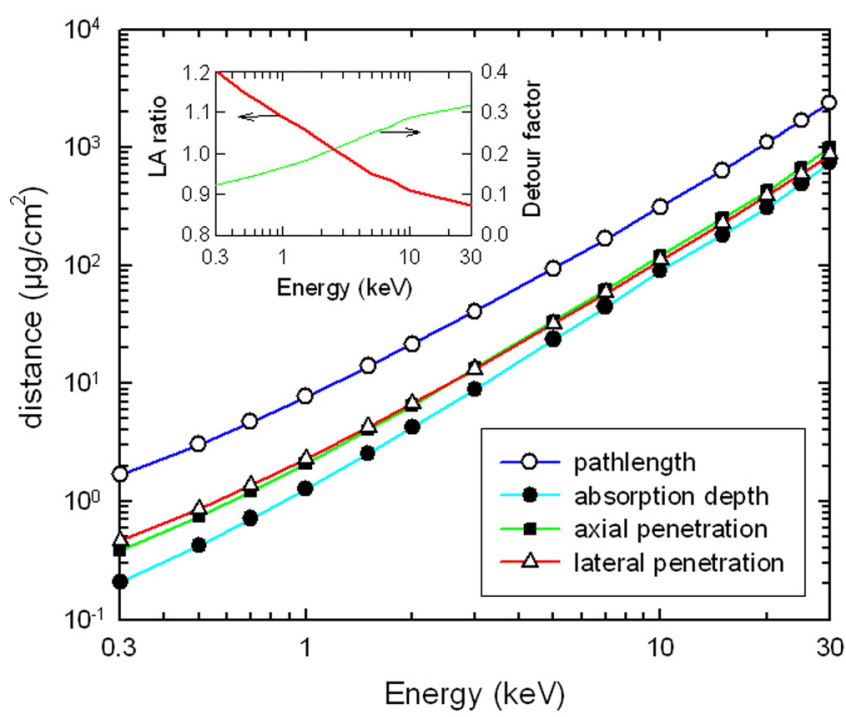

FIG. 2. (Color online) Monte Carlo simulations of the density-normalized pathlength $\left(\rho \bar{r}_{\text {total }}\right)$, absorption depth $\left(\rho \bar{z}_{n}\right)$, axial penetration $\left(\rho \bar{z}_{\max }\right)$, and lateral penetration $\left(\rho \bar{p}_{\max }\right)$ of an electron beam in MWCNT materials as a function of beam energy. The inset depicts the lateral-to-axial (LA) penetration ratio $\left(\frac{p_{\max }}{z_{\max }}\right)$ and the detour factor $\left(\frac{\bar{z}_{n}}{\bar{r}_{\text {total }}}\right)$. The lines are to guide the eye.

tion with, say, the $z$-direction and set the track starting point at $\left(x_{0}, y_{0}, z_{0}\right)=(0,0,0)$. Then, the electron absorption depth equals the average value of the $z$-coordinate of the final interaction point, $z_{n}$, (since $n$ designates the last collision) where the energy of the primary (beam) electron falls below the simulation cut-off. On the other hand, the maximum penetration depth along the incident beam direction (i.e. axially) is determined by the average value of $z_{\max }=\operatorname{Max}\left\{z_{i} ; i=1, \ldots, n\right\}$, where $z_{\max }$ is the interaction point with the largest $z$-coordinate. Note that, by definition, $z_{n}$ is always smaller (or equal) to $z_{\max }$. In fact, the two may differ considerably because at low energies and near the end of the track electrons undergo many large-angle deflections and will therefore tend to propagate back towards the origin. The maximum penetration depth perpendicular to the incident beam direction (i.e., laterally) is determined by the average value of $p_{\max }=\operatorname{Max}\left\{\left(x_{i}^{2}+y_{i}^{2}\right)^{1 / 2} ; i=1, \ldots, n\right\}$, where $p_{\max }$ is the interaction point with the largest radial distance from the incident beam direction. Note that $r_{\text {total }}, z_{n}, z_{\max }$, $p_{\max }$, refer to a single primary (beam) electron track while their average $\left(\bar{r}_{\text {total }}, \bar{z}_{n}, \bar{z}_{\max }, \bar{p}_{\text {max }}\right)$ is taken over the total number of beam electrons simulated.

The inset in Fig. 2 depicts the ratio between the lateral and axial penetration depths, $\frac{\bar{p}_{\max }}{\bar{z}_{\max }}$, herein referred to as the lateral-to-axial (LA) ratio, as well as the ratio between the electron absorption depth and pathlength, $\frac{\bar{z}_{n}}{\bar{r}_{\text {total }}}$, the so-called detour factor. ${ }^{38}$ Since the electron absorption depth (as defined above) measures the projection of the pathlength on the incident beam direction, the detour factor is a convenient measure of the "diminishing" effect that multiple scattering has upon the electron beam penetration capacity. As it can be seen from the inset of Fig. 2, the detour factor, although increases with beam energy, remains well below unity over the present energy range, indicating a significant deviation from a straight-line trajectory. On the other hand, the LA ratio starts from about 1.2 at $300 \mathrm{eV}$, reaches unity at about $3 \mathrm{keV}$, and diminishes to 0.87 at $30 \mathrm{keV}$. As an example, for 
a MWCNT material of $\rho=0.03 \mathrm{~g} / \mathrm{cm}^{3}$ (typical CNT forest density) we can deduce from Fig. 2 that an electron beam of $3 \mathrm{keV}$ will, on average, irradiate $4.44 \mu \mathrm{m}$ of material in both the axial and lateral beam directions, whereas for an electron beam of $30 \mathrm{keV}$ the corresponding irradiation depths are $334 \mu \mathrm{m} \quad$ (axially) and $334 \times 0.87=291 \mu \mathrm{m} \quad$ (laterally). Among other things, these results enable us to predict the irradiation volume (also called charged-carrier generation volume) as well as the spatial resolution in low-voltage SEM. $^{39}$

MC simulations of the energy dissipation profile (also called the depth-dose curve) in a MWCNT material including secondary-electron cascade generation have been carried out for several different electron beam energies in the interval $0.3-30 \mathrm{keV}$. The depth-dose curves are analytically represented by

$$
\frac{d T}{d z}=\frac{f T}{R} g(x),
$$

where $g(x)$ is the Everhart-Hoff function, ${ }^{40} R$ is the Gruen range, ${ }^{39,40}$ and $f$ is a normalization coefficient the value of which, although varying with beam energy, is $\sim 2$ to within a few $\%$ in the present energy range. The variable $x$ in $g(x)$ is the depth $z$ divided by $R$. The validity of Eq. (3), that is, of expressing the twice-normalized depth-dose curve, $\frac{(d T / T)}{(d z / R)}$, via the Everhart-Hoff function, $g(x)$, rests on the "high-energy" approximation that the large-angle scattering probability per unit fractional energy loss is insensitive to electron energy. ${ }^{40}$ Using a cubic polynomial approximation for $g(x)$, Eq. (3) has been applied to electron beam energies above $5 \mathrm{keV} .^{40,41}$ We here show that the utility of Eq. (3) can be extended to much lower energies using appropriate parameterizations for $g(x)$ and $R$ below and above $5 \mathrm{keV}$. Specifically, we approximate the Everhart-Hoff function by

$$
g(x)=\sum_{i=0}^{l} \alpha_{i} x^{i}
$$

with $l=3$ for $T \geq 5 \mathrm{keV}$ and $l=5$ for $T<5 \mathrm{keV}$ and coefficients, respectively, $\alpha_{0}=0.565183, \alpha_{1}=3.33252, \alpha_{2}$ $=-11.1593, \alpha_{3}=8.08361$ and $\alpha_{0}=0.752267, \alpha_{1}=9.55142$, $\alpha_{2}=-73.1166, \alpha_{3}=204.994, \alpha_{4}=-276.858, \alpha_{5}=148.602$. For use in Eqs. (3) and (4), we approximate $R$ by the simulated pathlength (see Fig. 2) which can be analytically represented (to within $\pm 5 \%$ ) by a Gruen-type formula $R=42.9 T^{1.865} \rho^{-1} \quad$ for $T>5 \mathrm{keV}$ and by $R=0.976$ $(2.05+11.3 T)^{1.684} \rho^{-1}$ for $T \leq 5 \mathrm{keV}$, with $R$ in nm, $T$ in $\mathrm{keV}$, and $\rho$ in $\mathrm{g} / \mathrm{cm}^{3}$. The above parameterization of the EverhartHoff function is valid up to depths $z=0.6 R$ which corresponds to $90 \%-99 \%$ of the dissipated beam energy in the material.

In Fig. 3 we present the mass thickness $(\rho z)$ of MWCNT materials where $20 \%\left(X_{20}\right), 50 \%\left(X_{50}\right)$, and $80 \%\left(X_{80}\right)$ of the beam energy is dissipated. The agreement between the MC simulations and the analytical predictions of Eq. (3) is fairly good over the entire energy interval studied $(0.3-30 \mathrm{keV})$. As expected, the agreement generally improves with increasing beam energy and energy dissipation fraction. Using the example of a MWCNT material of $\rho=0.03 \mathrm{~g} / \mathrm{cm}^{3}$, we can

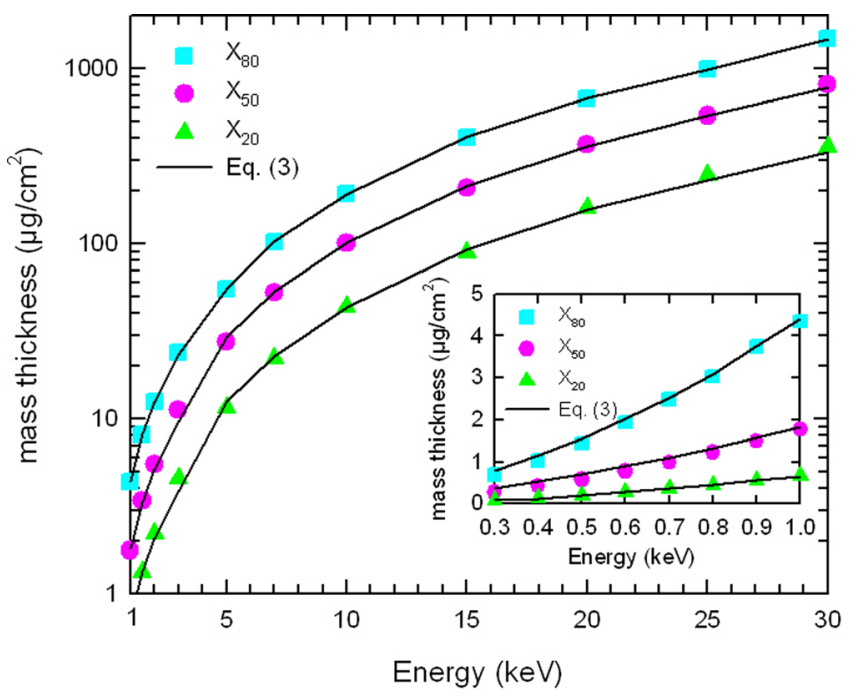

FIG. 3. (Color online) Mass thickness ( $\rho z)$ of MWCNT material where $20 \%$ $\left(\mathrm{X}_{20}\right), 50 \%\left(\mathrm{X}_{50}\right)$, and $80 \%\left(\mathrm{X}_{80}\right)$ of the beam energy is dissipated for electron-beam energies of $1-30 \mathrm{keV}$ (main panel) and $0.3-1 \mathrm{keV}$ (inset). Symbols are Monte Carlo simulations whereas the lines are analytical calculations using Eq. (3).

deduce from Eq. (3) that an electron beam of, say, $10 \mathrm{keV}$, will dissipate $80 \%$ of its energy within $64 \mu \mathrm{m}$ thickness of material, $50 \%$ within $33.4 \mu \mathrm{m}$ thickness, and $20 \%$ within $14.4 \mu \mathrm{m}$ thickness. It is also straightforward to determine the energy dissipation between any depths $z_{1}$ and $z_{2}$ in the irradiated material by computing the area under the depth-dose curve from Eq. (3).

To summarize, we have developed a quasi first-principles Monte Carlo model of energy dissipation by low-energy electron beams $(0.3-30 \mathrm{keV})$ in MWCNT materials using a dielectric response function description of the target electronic excitations. The model allows secondary-electron cascade generation to be explicitly simulated within a unified model of single-particle and plasmon excitations. Our results provide practical analytical functions for computing depth-dose curves and charged-carrier generation volumes for use in SEM/EBL irradiation effects studies in MWCNT materials.

Financial support for D.E., I.K., and K.K. by the European Union FP7 ANTICARB (HEALTH-F2-2008-201587) and for R.G.M. and I.A. by the Spanish Ministerio de Ciencia e Innovación (project FIS2010-17225) is acknowledged.

${ }^{1}$ A. V. Krasheninnikov and F. Banhart, Nat. Mat. 6, 723 (2007).

${ }^{2}$ A. V. Krasheninnikov and K. Nordlund, J. Appl. Phys. 107, 071301 (2010).

${ }^{3}$ K. Kanzaki, S. Suzuki, H. Inokawa, Y. Ono, A. Vijayaraghavan, and Y. Kobayashi, J. Appl. Phys. 101, 034317 (2007).

${ }^{4}$ G. Rius, J. Llobet, M. J. Esplandiu, L. Sole, X. Borrise, and F. Perez-Murano, Microelectr. Eng. 86, 892 (2009).

${ }^{5}$ J. Chan, B. Burke, M. Cabral, C. Hu, J. Campbell, L. Harriott, and K. A. Williams, J. Phys.: Condens. Matter 22, 334212 (2010).

${ }^{6}$ A. Nojeh, W.-K. Wong, E. Yieh, and R. F. Pease, J. Vac. Sci. Technol. B 22, 3124 (2004).

${ }^{7}$ M. Michan, P. Yaghoobi, B. Wong, and A. Nojeh, Phys. Rev. B 81, 195438 (2010).

${ }^{8}$ M. K. Alam, P. Yaghoobi, and A. Nojeh, Scanning 31, 221 (2010).

${ }^{9}$ J. Luo, J. H. Warner, C. Feng, Y. Yao, Z. Jin, H. Wang, C. Pan, S. Wang,

L. Yang, Y. Li, J. Zhang, A. A. R. Watt, L.-M. Peng, J. Zhu, and G. A. D.

Briggs, Appl. Phys. Lett. 96, 213113 (2011). 
${ }^{10}$ D. C. Joy, Monte Carlo Modeling for Electron Microscopy and Microanalysis (Oxford University Press, New York, 1995).

${ }^{11}$ M. Dapor, Electron-Beam Interactions with Solids: Application of the Monte Carlo Method to Electron Scattering Problems (Springer, New York, 2003).

${ }^{12}$ B. W. Smith and D. E. Luzzi, J. Appl. Phys. 90, 3509 (2001).

${ }^{13}$ A. V. Krasheninnikov, F. Banhart, J. X. Li, A. S. Foster, and R. M. Nieminen, Phys. Rev. B 72, 125428 (2005).

${ }^{14}$ A. V. Krasheninnikov and K. Nordlund, Nucl. Instrum. Meth. B 216, 355 (2004).

${ }^{15}$ M. K. Alam, P. Yaghoobi, and A. Nojeh, J. Vac. Sci. Technol. B 28, C6J13 (2010).

${ }^{16}$ M. K. Alam and A. Nojeh, J. Vac. Sci. Technol. B 29, 041803 (2011).

${ }^{17}$ W. S. M. Werner, Surf. Interface Anal. 31, 141 (2001).

${ }^{18}$ Y. Chihara, M. Yasuda, S. Wakuda, H. Kawata, and Y. Hirai, J. Vac. Sci. Technol. B 29, 06FG09 (2011).

${ }^{19}$ M. Yasuda, R. Mimura, H. Kawata, and Y. Hirai, J. Appl. Phys. 109, 054304 (2011).

${ }^{20}$ R. Shimizu and Z.-J. Ding, Rep. Prog. Phys. 55, 487 (1992).

${ }^{21}$ Y.-N. Wang and Z. L. Miskovic, Phys. Rev. A 66, 042904 (2002).

${ }^{22}$ A. Balassis and G. Gumbs, Phys. Rev. B 74, 045420 (2006).

${ }^{23}$ D. J. Mowbray, S. Segui, J. Gervasoni, Z. L. Miskovic, and N. R. Arista, Phys. Rev. B 82, 035405 (2010).

${ }^{24}$ A. G. Marinopoulos, L. Reining, and A. Rubio, Phys. Rev. B 78, 235428 (2008).

${ }^{25}$ C. J. Powell and A. Jablonski, J. Phys. Chem. Ref. Data 28, 19 (1999).

${ }^{26} \mathrm{~S}$. Tougaard and F. Yubero, Surf. Interface Anal. 36, 824 (2004).
${ }^{27}$ S. Tanuma, C. J. Powell, and D. R. Penn, J. Appl. Phys. 103, 063707 (2008).

${ }^{28}$ S. Tanuma, C. J. Powell, and D. R. Penn, Surf. Interface Anal. 43, 689 (2011).

${ }^{29}$ I. Kyriakou, D. Emfietzoglou, R. Garcia-Molina, I. Abril, and K. Kostarelos, Appl. Phys. Lett. 94, 263113 (2009).

${ }^{30}$ I. Kyriakou, D. Emfietzoglou, R. Garcia-Molina, I. Abril, and K. Kostarelos, J. Appl. Phys. 110, 054304 (2011).

${ }^{31}$ Z. L. Wang, Micron 27, 265 (1996).

${ }^{32}$ M. Inokuti, Rev. Mod. Phys. 43, 297 (1971).

${ }^{33}$ D. Emfietzoglou, I. Kyriakou, R. Garcia-Molina, I. Abril, and K. Kostarelos, J. Appl. Phys. 108, 054312 (2010).

${ }^{34}$ R. Browning, Appl. Phys. Lett. 58, 2845 (1991).

${ }^{35}$ A. Jablonski, F. Salvat, and C. J. Powell, NIST Electron Elastic-Scattering Cross-Section Database Version 3.1, National Institute of Standards and Technology, Gaithersburg, MD, 2003.

${ }^{36}$ F. Salvat, A. Jablonski, and C. J. Powell, Comp. Phys. Comm. 165, 157 (2005).

${ }^{37}$ W. S. M. Werner, F. Salvat-Pujol, W. Smekal, R. Khalid, F. Aumayr, H. Störi, A. Ruocco, and G. Stefani, Appl. Phys. Lett. 99, 184102 (2011).

${ }^{38}$ International Commission on Radiation Units and Measurements (ICRU), ICRU Report 49, 1993, Bethesda, MD.

${ }^{39}$ O. Kurniawan and V. K. S. Ong, Scanning 29, 280 (2007).

${ }^{40}$ T. E. Everhart and P. H. Hoff, J. Appl. Phys. 42, 5837 (1971).

${ }^{41}$ H. Nykänen, P. Mattila, S. Suihkonen, J. Riikonen, E. Quillet, E. Homeyer, J. Bellessa, and M. Sopanen, J. Appl. Phys. 109, 083105 (2011). 